Int. J. Dev. Biol. 64: 353-366 (2020)

https://doi.org/10.1387/ijdb.190130sg

\title{
Neural cells and their progenitors in regenerating zebrafish spinal cord
}

\author{
SUBHRA PRAKASH HUI", ${ }^{\# A P A S ~ C H A N D R A ~ N A G ~}{ }^{2}$, SUKLA GHOSH ${ }^{*, 1}$ \\ ${ }^{1}$ Department of Biophysics, Molecular Biology and Bioinformatics, University of Calcutta, Kolkata, India and \\ ${ }^{2}$ Department of Anatomy, All India Institute of Medical Sciences, New Delhi, India
}

\begin{abstract}
The zebrafish (Danio rerio), among all amniotes is emerging as a powerful model to study vertebrate organogenesis and regeneration. In contrast to mammals, the adult zebrafish is capable of regenerating damaged axonal tracts; it can replace neurons and glia lost after spinal cord injury (SCl) and functionally recover. In the present paper, we report ultrastructural and cell biological analyses of regeneration processes after SCl. We have focused on event specific analyses of spinal cord regeneration involving different neuronal and glial cell progenitors, such as radial glia, oligodendrocyte progenitors (OPC), and Schwann cells. While comparing the different events, we frequently refer to previous ultrastructural analyses of central nervous system (CNS) injury in higher vertebrates. Our data show (a) the cellular events following injury, such as cell death and proliferation; (b) demyelination and remyelination followed by target innervation and regeneration of synaptic junctions and c) the existence of different progenitors and their roles during regeneration. The present ultrastructural analysis corroborates the cellular basis of regeneration in the zebrafish spinal cord and confirms the presence of both neuronal and different glial progenitors.
\end{abstract}

KEY WORDS: regeneration, spinal cord, zebrafish, astroglia, neural progenitor

\section{Introduction}

Teleost fish like Danio rerio are unique among the adult vertebrates in their capacity to regenerate lost or damaged parts of body including their central nervous system (CNS). Spinal cord injury (SCl) in mammals leads to decline in various functions because of progressive patho-physiological disturbances which cause secondary injury (Balentine, 1978a, b; Blight, 1985; Popovitch et al., 1997; Guth et al., 1999). Both acute and secondary injury of spinal cord involves complex cellular and molecular mechanisms which eventually lead to permanent irreparable damages to the cord and resultant functional deficit. In contrast to mammals, zebrafish can replace its lost cells, both neuron and glia, repair its severed axons with appropriate target innervations, thus achieve functional recovery after an injury. In this context, adult zebrafish represents an excellent model to study CNS regeneration addressing all different cellular aspects of this complex process and provide more insights that may be extrapolated to induce regeneration in higher vertebrates.

The primary requirements for successful regeneration to occur after SCI include: replenishment of lost cells which involve glia and neuron, survival and re-growth of severed axons, remyelination and appropriate target innervations of the regenerating axons. In order to understand the similarities and differences in the injury response between mammalian and zebrafish spinal cord, we undertook ultrastructural analysis which may further strengthen our understanding of cellular and molecular basis of regeneration. Early injury response in zebrafish spinal cord includes infiltration of blood cells predominantly macrophages, cell death and demyelination, which are followed by injury induced proliferation and neurogenesis. The late response after injury appears to be axonal re-growth and appropriate target innervations (Hui et al., 2010). Previous studies on teleost fish also suggested that proliferation and neurogenesis can occur in different areas of zebrafish brain

Abbreviations used in this paper: $\mathrm{BBB}$, blood brain barrier; BLBP, brain lipid binding protein; CNS, central nervous system; GFAP, glial fibrillary acidic protein; GLAST, glutamate aspartate transporter; NSC, neural stem cell; OPC, oligodendrocyte progenitor; qRT-PCR, quantitative reverse transcriptase-polymerase chain reaction; RER, rough endoplasmic reticulum; SCI, spinal cord injury.

\footnotetext{
*Address correspondence to: Sukla Ghosh. Department of Biophysics, Molecular Biology and Bioinformatics, University of Calcutta, Kolkata, India.

E-mail: suklagh2010@gmail.com
}

\#Present address: S.N. Pradhan Centre for Neurosciences, University of Calcutta, 35 Ballygunge Circular Road, Kolkata 700019, India.

Supplementary Material ( 1 table +3 figures) for this paper is available at: https://doi.org/10.1387/ijdb.190130sg

Submitted: 25 June, 2019. Accepted: 26 July, 2019.

ISSN: Online 1696-3547, Print 0214-6282

(1) 2020 UPV/EHU Press

Printed in Spain 
and spinal cord (Zupanc et al., 2005; Adolf et al., 2006; Reimer et al., 2008; Hui et al., 2010; Ito et al., 2010). Proliferating progenitor cells are also present in different compartments of CNS similar to the neural stem cells (NSC) in mammalian CNS (Johansson et al., 1999; Temple and Alvarez-Buylla, 1999). We observe presence of different proliferating progenitors which are contributing to regeneration of zebrafish spinal cord. The spatio-temporal analysis of these progenitors (both neuronal and glial progenitors) in regenerating spinal cord have been elucidated, as these cells are identified by ultrastructural characters and by using appropriate immunohistological markers.

Any injury to the mammalian CNS induces glial scar formation and because of the fibro-astrocytic scar axons cannot regenerate beyond the lesion. The reactive astrocytes in the glial scar play a prominent role in regeneration failure (Rugde and Silver, 1990; Silver and Miller, 2004) although some evidence exists in favor of a beneficial role of glial scar (Faulkner et al., 2004). Reactive glia may form an extra barrier to severed axons after $\mathrm{SCl}$ and it is not just the scar but other cell types may be potentially involved to exert more deleterious effect (Silver, 2016). The role of glial scar and astrocyte is increasingly becoming a complex issue to untangle as reactive glia express markers associated with neurogenic stem cells (Silver and Steindler, 2009) and their stem cell like properties can be recognized both in vitro and in vivo (Buffo et al., 2008).

Until now, comprehensive ultrastructural characterization of cellular composition in adult zebrafish spinal cord pre-injury and their response to $\mathrm{SCI}$ has not been studied in its entire gamut. In the present study, we aimed to unravel the ultrastructural characteristics of different cellular events and identify associated cells involved in regeneration of zebrafish spinal cord in order to understand the underlying cellular basis of regeneration. Astroglial response is one of the most important aspects of differential outcome after CNS injury in mammals and fish. We have analyzed some of the features of these cells like glial hypertrophy, astroglia- neuron interaction by electron microscopy and immunohistological markers. We also found some of these progenitors are important contributors to the successful regeneration in this species. Our time course analysis have demonstrated some characteristic cellular events which are unique to zebrafish spinal cord while some other cellular events are common to both mammalian and zebrafish cord. The present ultrastructural analysis also corroborates with the cellular and molecular features shown in zebrafish cord before and after injury (Becker and Becker, 2001; Reimer et al., 2008; Hui et al., 2010, 2015; Ohnmacht et al., 2016).

\section{Results}

\section{Cellular organization of the uninjured adult zebrafish cord}

The anatomy of normal uninjured adult spinal cord in zebrafish has already been characterized using different histological staining (Hui et al., 2010). Our present ultrastructural analyses of uninjured cord reveal the presence of bundles of both myelinated as well as unmyelinated axons in the white matter. Within the axoplasm, there are mitochondria and neurofilaments (Fig. 1A) and myelinated axons show compact array of myelin sheath (Fig. $1 \mathrm{~A}$ inset). The oligodendrocytes possess electron dense nucleus with aggregates of heterochromatin (Fig. 1B) and are present in the white matter. They also show some of the typical features
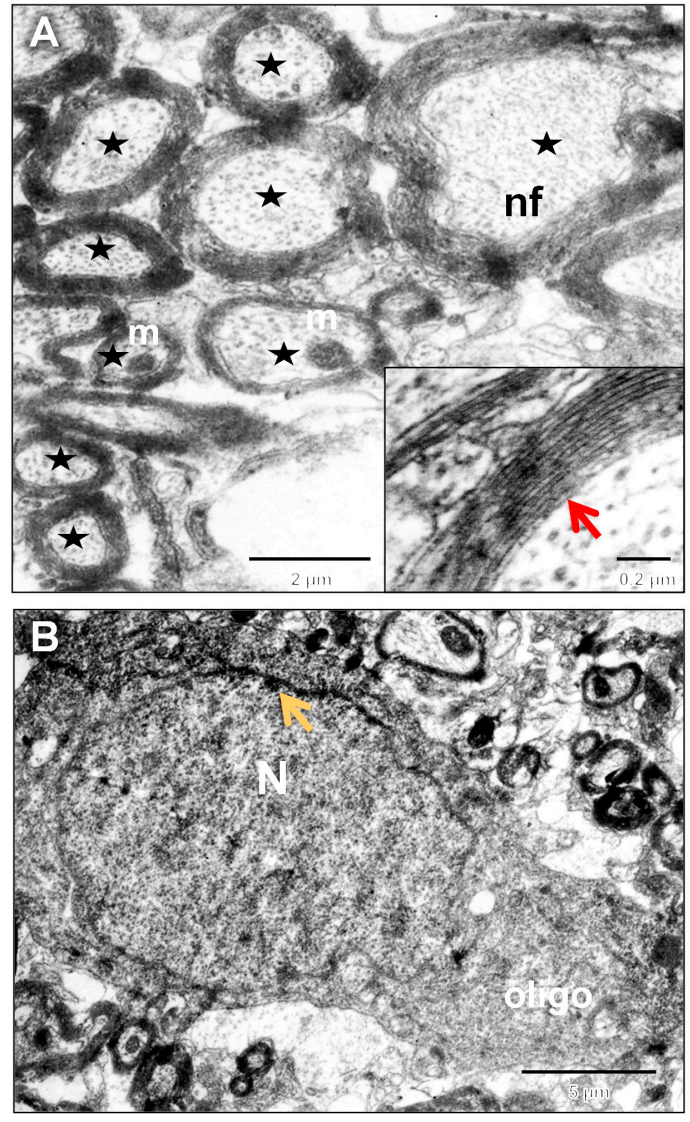
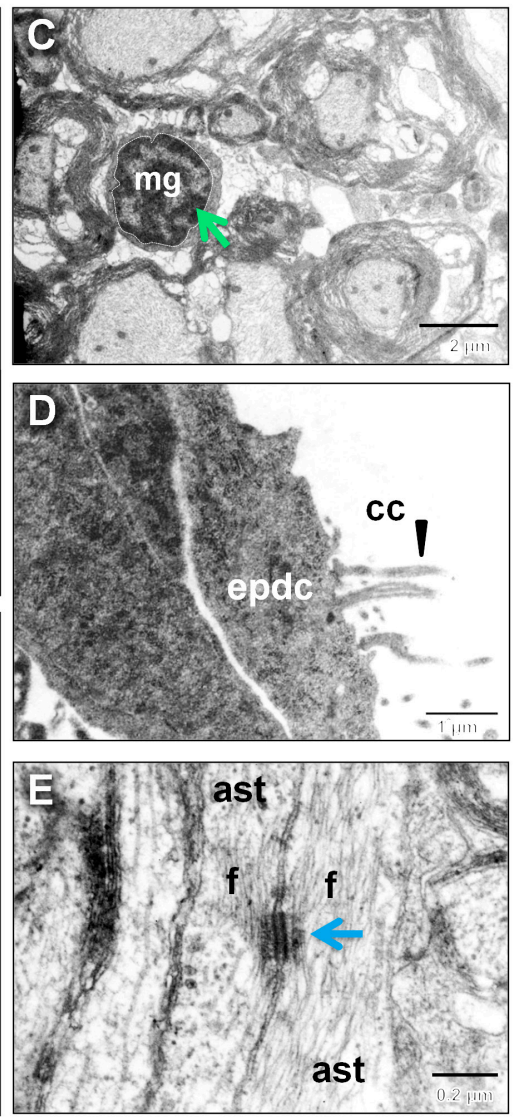

Fig. 1. Ultrastructural characterization of uninjured zebrafish spinal cord. (A) Transverse section of the white matter region of an uninjured spinal cord shows many myelinated axons (stars). The cytoplasm of axons contains neurofilaments (nf) and cell organelles like mitochondria $(m)$. The inset shows the fine structure of myelin sheath (red arrow) in higher magnification. (B) TEM image shows a mature oligodendrocyte (oligo), showing nucleus (N) and peripheral heterochromatin (orange arrow) present in the white matter of spinal cord. (C) TEM image of spinal cord white matter shows the presence of microglia ( $\mathrm{mg}$ ) with little cytoplasm surrounding the nucleus (white dotted circle) and heterochromatin (green arrow). (D) Transverse section through the ependymal region, showing an ependymal cell (epdc) with characteristic microvilli (arrowhead) extended towards the central canal (CC). (E) TEM image shows the junction between two astroglia (ast) connected through desmosome (blue arrow). Note that the cytoplasm of both astrocytes is filled with intermediate filaments (f). Scale bar, 2 $\mu \mathrm{m}(A) 5 \mu \mathrm{m}(B), 2 \mu \mathrm{m}$ (C), $1 \mu \mathrm{m}$ (D), $0.2 \mu \mathrm{m}$ (E). 

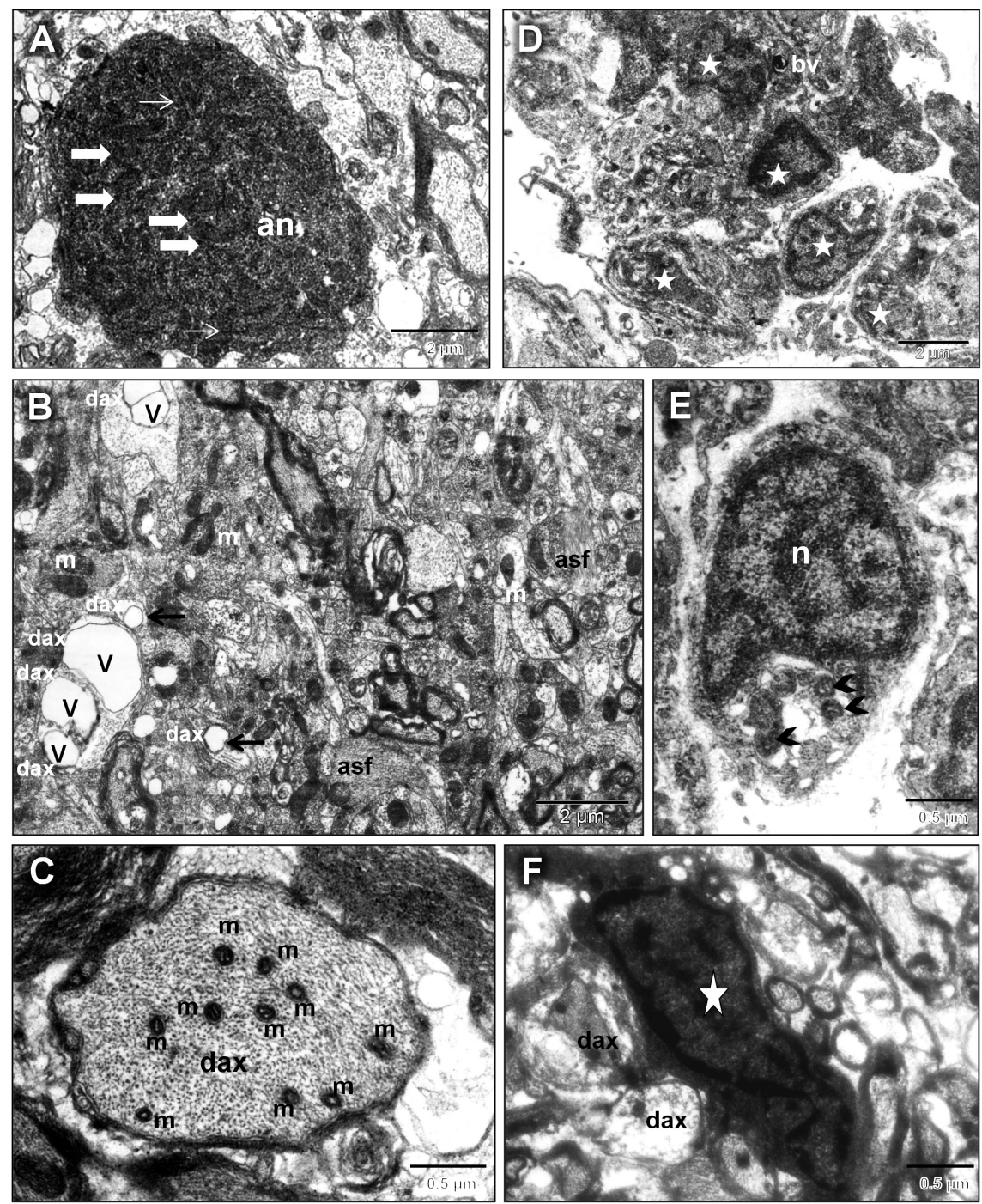

Fig. 2. Ultrastructural characteristics of early response to spinal cord injury. (A) TEM image shows the cytoplasm of an apoptotic neuron in a 3 dpicord with dark irregular mitochondria (thin arrows), fragmented nucleus (thick arrows) and retracted cytoplasm (adapted from Hui etal., 2010). (B) TEM image of injury epicenter of a 3 dpi cord showing many degenerated axons (dax) with a thin covering of myelin sheath (black arrow). Presence of mitochondria (m) within degenerated axons and hypertrophic astroglial filaments (asf) are obvious. Many void spaces marked as $V$ are present within degenerated axons. (C) Transverse section of 3 dpi cord showing densely packed neurofilaments (fine black dots) and many mitochondria ( $\mathrm{m}$ ) in the cytoplasm of a denuded axon (dax). (D) TEM image of 3 dpi spinal cord section showing disorganized tissue in the injury epicenter, accumulation of multiple phagocytic cells (white stars), and an adjacent blood vessel (bv) that is spared after injury. (E) TEM image in higher magnification shows a microglial cell with engulfed myelin debris (arrowheads) and numerous vacuoles in its cytoplasm. ' $n$ ' denotes the nucleus of the microglial cell. (F) A phagocytic macrophage (star) in the vicinity of several degenerating axons (dax) in a 3 dpi cord. Scale bar, 2 $\mu \mathrm{m}(A, B$ and $D) ; 0.5 \mu \mathrm{m}(C, E$ and $F)$. like short rough endoplasmic reticulum (RER) and microtubules in the cytoplasm, as described (Peters et al., 1991). Other glial components like resident microglia can also be observed. These cells have scanty cytoplasm and large nucleus with peripheral heterochromatin and the heterochromatin network is made up of many fused granules as represented in Fig. 1C. The grey matter volume in zebrafish cord is relatively less compared to that of mammals. In the central region of the cord runs the central canal or ependymal canal which is surrounded by ependymal cells. Neurons are present in the subependymal region (data not shown). The ependymal cells (Fig. 1D) are characterized by the presence of extended processes or cilia facing towards the lumen of the central canal. Some of the ependymal cells are radial glial progenitors, as these cells are GFAP positive and soma of them are facing towards central canal and extending branched fibers towards the pial membrane (Supplementary Figure 1). These glias are heterogeneous as different subpopulations can be identified based on expression of combinations of markers such as GLAST, BLBP and GFAP (Hui et al., 2015, Supplementary Figure 2). We also observe desmosomes between the processes of astroglial cells which have electron lucent cytoplasm with glial filaments (Fig. $1 \mathrm{E})$, suggesting the presence of well developed glial networks in uninjured adult zebrafish cord. Most of the astroglia that we could identify in grey matter are actually radial glia rather than mature astrocytes. Thus, astroglias were identified ultrastructurally by the presence of moderate content of intermediate filaments in their cytoplasmic processes and few dark inclusions in their cell bodies. Their nuclei appeared to be homogeneous in chromatin content. Oligodendrocytes were identified by the presence of a large nucleus, few short cisternae of rough endoplasmic reticulum and microtubules in their broad processes, while microglia appeared with several processes and few lysosome-like granules in their cytoplasm, as described by Peters et al., (1981). In normal uninjured spinal cord, distinct ultrstructural characters of different components are shown in the figure and mentioned in Supplementary Table 1.

\section{Early cellular responses to injury}

Following $\mathrm{SCl}$, there is immediate disruption of blood brain barrier (BBB) leading to infiltration of blood cells in the injury epicenter. Other than neutrophils and monocytes, the predominant variety of wandering cells present near the wound site is macrophages or microglia. Early cellular response to injury is very dynamic; it involves the loss of BBB, followed by glial and neuronal loss, and fragmentation of myelin of injured axons, which occur within 2-3 
Fig. 3. Glial and neuronal progenitors in injured spinal cord. (A) Longitudinal section of a 7 dpi spinal cord immunostained both with Sox2 and BrdU, showing many proliferating progenitors (white arrows). Nuclei are stained with DAPI. (B,C) Transverse section of a $7 \mathrm{dpi}$ spinal cord immunostained with Sox2 and vimentin, shown in low (B) and high (C) magnification. Nuclei are stained with DAPI. (C1-C4) The higher magnification view of yellow boxed area in $C$ shows a Sox2 and vimentin colocalized glial progenitor (yellow arrow) represented as merge and all individual channels. (D,E) Transverse section of a 7 dpi spinal cord immunostained with Sox2 and HuC/D in low (D) and high (E) magnification. Nuclei are stained with DAPI. (E1-E4) The higher magnification view of yellow boxed area in E shows a Sox2 and HuC/D colocalized neuronal progenitor (orange arrow) around the ependymal canal (epc) represented as merge and all individual channels. Scale bars, $100 \mu \mathrm{m}(A) ; 50 \mu \mathrm{m}(B-E) ; 20 \mu \mathrm{m}$ (C1-C4, E1-E4).
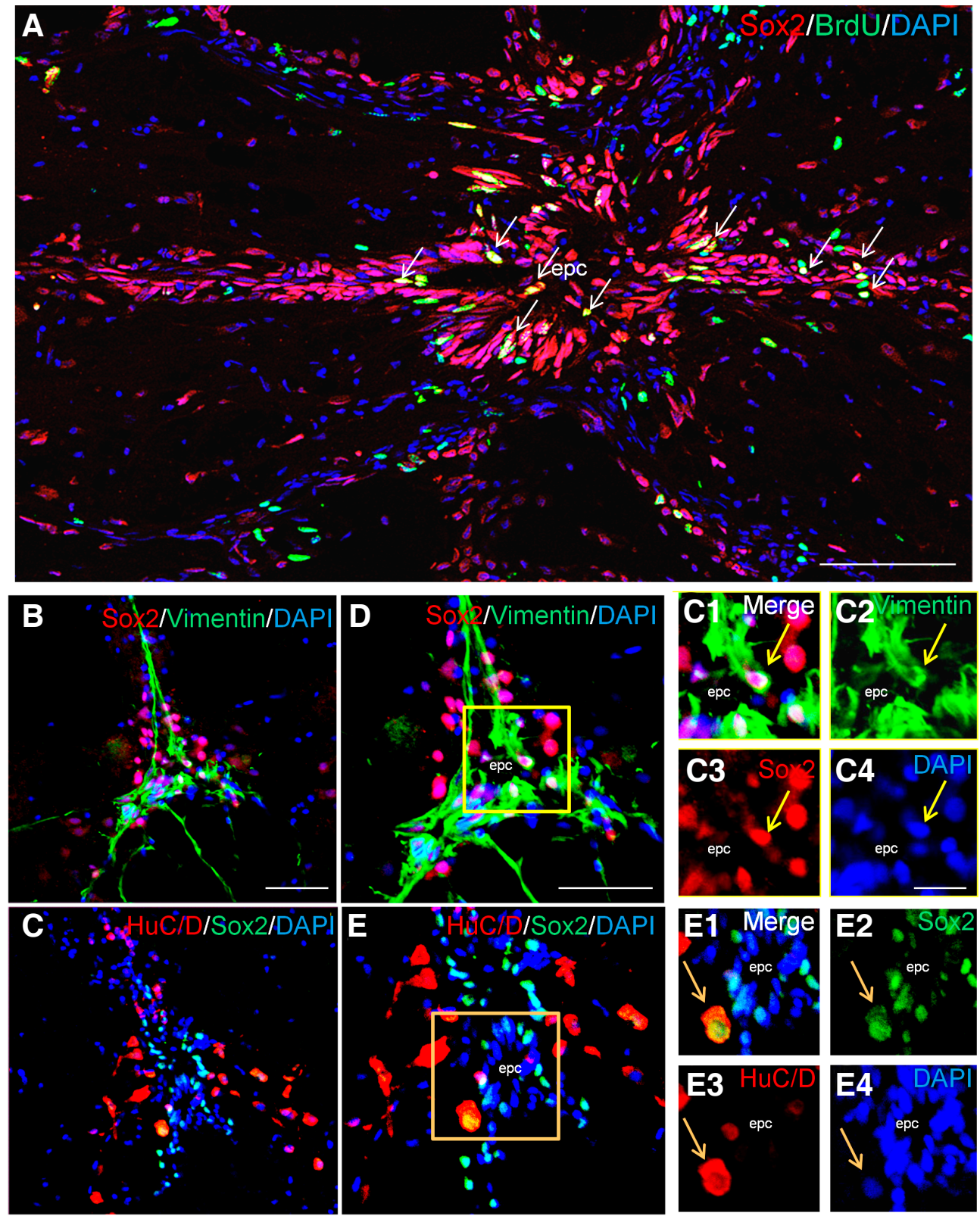

days of injury. Dying neurons with fragmented nucleus can be seen in the injury epicenter, as shown in a 3 day post injury (dpi) cord (Fig. 2A). The injury epicenter shows inflammation and degenerative changes, and that may continue for few more days after initial response. The injury epicenter is characterized by presence of many degenerating axons, swollen mitochondria, void structures within many axonal cytoplasm and astroglial filaments near denuded axons (Fig. 2B). The damaged axons undergo sloughing off myelin sheaths as a consequence of injury. In these injured axons, increased presence of neurofilaments and mitochondria are also evident (Fig. 2 B,C). Dense distribution of neurofilaments and many swollen mitochondria within axonal cytoplasm of the injured axons as well as presence of hypertrophic astroglial processes are all associated with early injury response.

Injury of spinal cord disrupts the BBB and allows blood cells to infiltrate into the lesion. The infiltration of blood-borne macrophages is evident quite early in 2-3 dpi cord and these cells are phagocytic in nature (Fig. $2 \mathrm{D}, \mathrm{E}$ ). The damaged axons show breakdown of myelin sheaths. The phagocytic macrophages are responsible for engulfing axonal debris (Fig. 2E, F) and often show lipid filled vacuoles.

\section{Proliferative response and progenitors}

Early injury response is followed by rapid cellular migration and proliferative response as BrdU incorporation starts in $3 \mathrm{dpi}$ cord, peaks in $7 \mathrm{dpi}$ cord, which gradually decreases at later time points of injury (Hui et al., 2010). Many of the ependymal cells are proliferating progenitor(s) and identified as radial glia (Supplementary Fig. 2), as they express various markers of radial glia. High expression of many of neural progenitor markers is also shown in $7 \mathrm{dpi}$ cord where rate of proliferation is known to be highest. The relative fold change of expressions of all these markers in 
$7 \mathrm{dpi}$ cord are significantly higher than uninjured cord, suggests increased proliferative response after injury and presence of proliferative progenitors (Fig. 3; Supplementary Fig. 3). The detailed ultrastructural characters of these progenitors are shown here.

\section{Neuronal progenitors}

Proliferative response of radial glia may lead to neurogenesis as previously reported in many studies. We corroborate our ultrastructural analysis and immunohistochemical analysis showing presence of neural progenitors by using different neuronal markers like Sox2, nestin, vimentin and HuC/D (Figs. 3, 4, and Supplementary Fig. 3). We have also carried out quantitative analysis of neural progenitor markers by quantitative reverse transcriptase-polymerase chain reaction (qRT-PCR) (Supplementary Fig. 3) and indeed among these Sox2/BrdU positive proliferating progenitors (Fig. 3A) some are neuronal progenitors since these cells are co-labeled with Sox2/ HuC/D (Fig. 3D). Ultrastructurally, we confirm the presence of newly born neuronal cells in $7 \mathrm{dpi}, 10 \mathrm{dpi}$ cord (Fig. 4 A,B,C) and even in 1 month regenerate showing immature neuron with high nucleo/ cytoplasmic (N/C) ratio with less elaborate cytoplasm having few organelles. While the newly born neurons become more and more mature, they develop characteristic organelles like rosette shaped free ribosome (Fig. 4A.1), well developed yet variably shaped mitochondria (Fig. 4A.1,C.1,C.2) and lysosomes (Fig. 4C.3) within the neuronal cytoplasm in the regenerates. There are neuronal progenitors in the grey matter around the ependyma associated with glial filaments in 10 dpi cord (Figs. 4D, 5F). We see vertically migrating elongated cells closely apposed with glial fibres in many occasion, particularly after proliferation and during neurogenesis.

\section{Glial progenitors}

The proliferating progenitor cells in a regenerating cord are of different types as there exist both neuronal (Figs. 3 D,E and 4) and glial progenitors (Fig. 5, Supplementary Figure 2). Fig. 5A shows ultrastructure of an early glial progenitor with dark nucleus and very little cytoplasm in the white matter of $10 \mathrm{dpi}$ cord. Glial progenitor of probable astrocytic lineage can be seen in Fig. 5A, B. The cell has a large nucleus and less cytoplasm (high N/C ratio) with
Fig. 4. Ultrastructural characteristics of newly born neuronal cells after injury in the zebrafish spinal cord. (A) A newly born neuron, with nucleus ( $n$ ) and cytoplasm (c) in 7 dpicord section. (A.1) High magnification view of $(A)$ showing organelles like mitochondria $(m)$ and ribosomes ( $r$ ). (B) An immature neuron with a large nucleus (n) and few organelles in the cytoplasm (c) in a $10 \mathrm{dpi} \mathrm{cord.} \mathrm{(C)} \mathrm{An} \mathrm{immature}$ neuron (triangle) with a large nucleus (n) with little cytoplasm (c) having fewer organelles and a newly born yet specified neuron lasterisk) with higher number of organelles and a blood vessel nearby (bv) in a $10 \mathrm{dpi}$ cord. The cytoplasm (c) of the specified neuron (star) is showing organelles and a large nucleus of the same neuron marked as ' $n$ '. (C.1-C.2) High magnification images showing organelles like mitochondria (m), Golgi bodies (g), ribosomes (r) within the cytoplasm of neurons in $10 \mathrm{dpi}$ cord. (C.3) Accumulation of lysosomes (arrows) in a neuron of 1 month old regenerated cord, the adjacent neuronal axon terminal shows immature synaptic vesicles (arrowheads). (D) A representative picture of proliferating neurons along with associated glial fibres with plenty of astrocytic filaments (f) in a regenerating 10 dpi cord. Note at least four elongated cells and their close association with glial fibres present near the ependyma. ' $n$ ' denotes nucleus of the proliferating neurons. Scale bars, $1 \mu \mathrm{m}$ ( $A$ and B); $0.2 \mu \mathrm{m}$ (A. 1); $5 \mu \mathrm{m}(C) ; 0.5 \mu \mathrm{m}$ (C. 1, C.2 and C.3); $2 \mu \mathrm{m}(\mathrm{D})$.
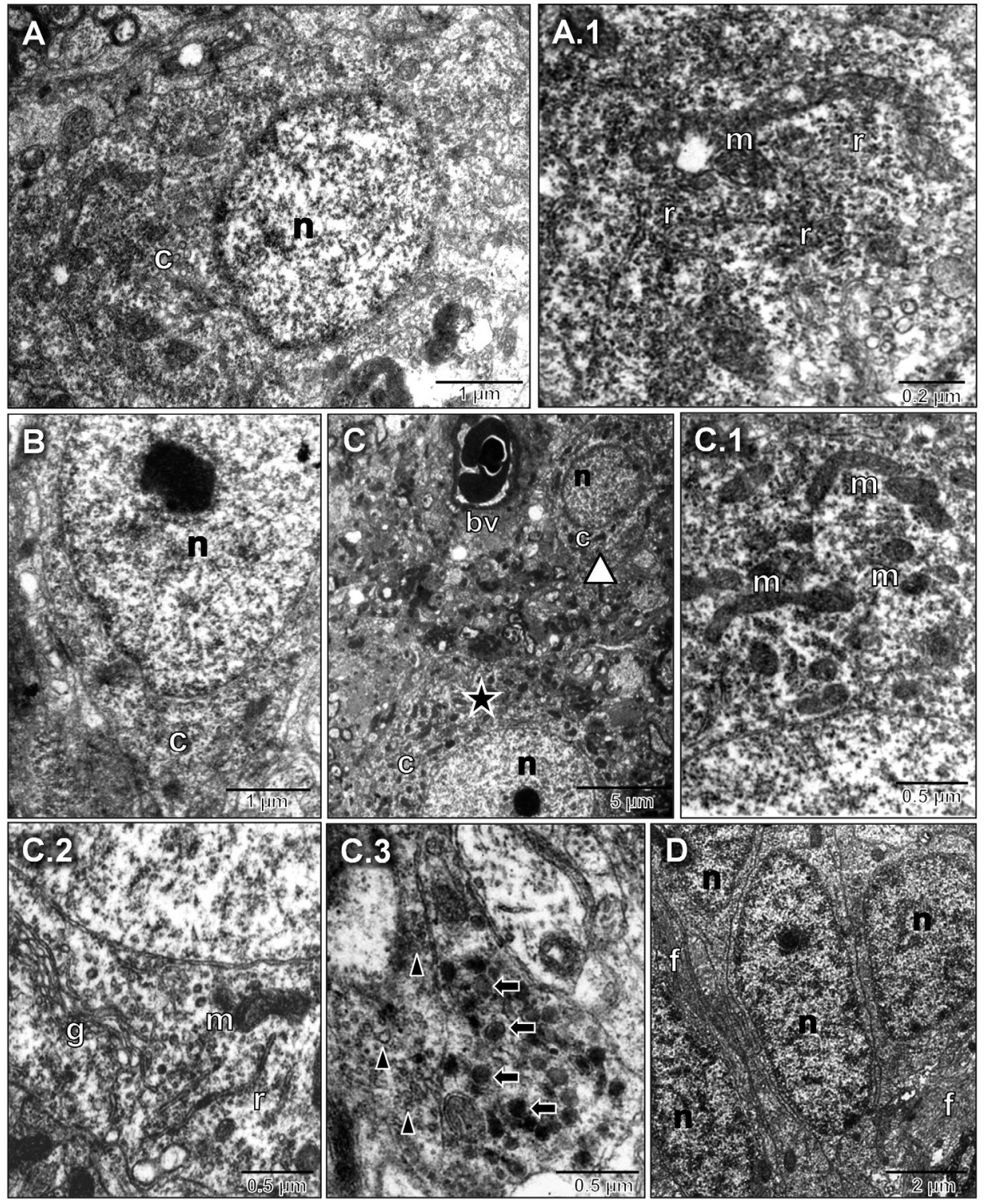

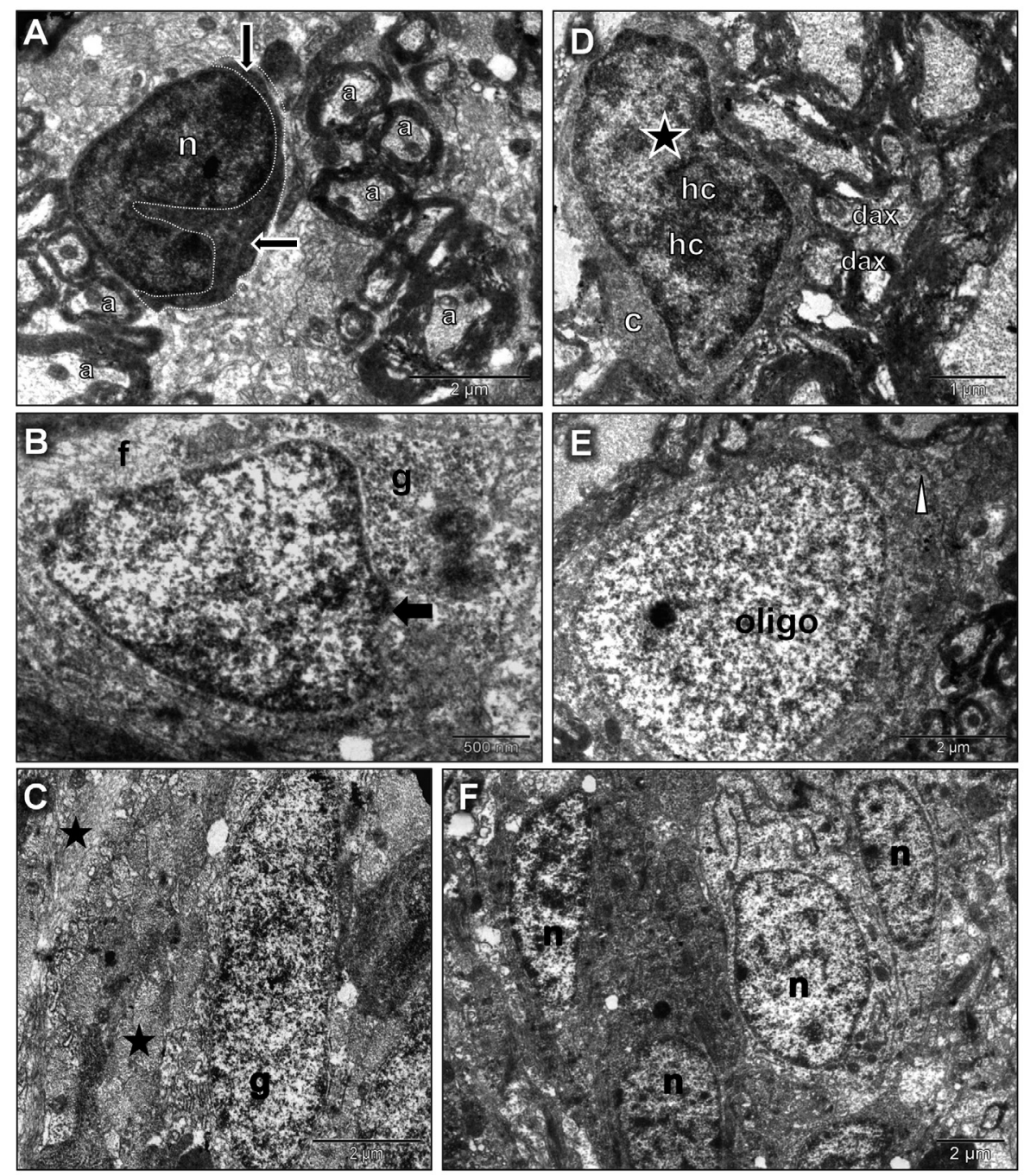

Fig. 5. Ultrastructural characteristics of different glial progenitors after injury in the zebrafish spinal cord. (A) TEM image showing several axons (a) and an early glial progenitor cell having a dark nucleus (n) and scanty cytoplasm (arrow) in a 7 dpi cord. The white line demarcates the boundary of nucleus (n). (B) An immature astrocyte (black arrow) showing intermediate filaments (f) and glycogen granules (g) in the cytoplasm. (C) Section from 10 dpi cord. Another representative picture of an immature glial cell $(g)$ in the white matter showing glial processes (stars). (D) An immature oligodendrocyte (star) with clumps of heterochromatin (hc) and scanty cytoplasm (c) surrounded by demyelinating axons (dax). (E) An oligodendrocyte (oligo) with semi-differentiated cytoplasm with few short cisternae of RER (white arrowhead). (F) 10 dpi cord section shows proliferating, neuronal precursor cells ( $n$ ) in the grey matter of the cord. Scale bar, $2 \mu \mathrm{m}$ (A, C, E and F); $1 \mu \mathrm{m}$ (D); $500 \mathrm{~nm}$ (B). glycogen granules and intermediate filaments (Fig. 5B). It is an immature astroglial cell, and we do not see mature astrocytes in the white matter. Similarly, few immature oligodendrocytes can be seen; these cells contain typical clumps of heterochromatin in the large nucleus, peripheral heterochromatin near nuclear membrane and small volume of cytoplasm. Small cisternae of RER are also visible in the cytoplasm of the immature oligodendrocytes (Fig. $5 \mathrm{D}, \mathrm{E})$. The particular immature oligodendrocyte is seen in the vicinity of few demyelinated axons in white matter (Fig. 5D) in a $10 \mathrm{dpi}$ cord. The predominant glial progenitor in zebrafish is radial glia (Hui et al., 2010), which is ependymoglia and can acquire bipolar shape after injury (Goldshmit et al., 2012). They appropriately express multiple glial markers like GLAST/BLBP/GFAP (Supplementary Figs. 1, 2, Hui et al., 2015) and also coexpress progenitor markers, Sox2 and vimentin (Fig. 3). Moreover, qRTPCR analysis showed the upregulation of intermediate filament genes gfap, vim and nes at 7 dpi cord (Supplementary Fig. 3), when we see highest rate of progenitor proliferation. Although, the heterogeneity is observed with radial glia (Supplementary Fig. 2), however, the contributions of the particular radial glial subpopulation with respect to their specific functions have not been studied.

\section{Response of astrocytic glia to injury}

Astrocytes are a particular type of glial cells in CNS and are remarkably complex. Astrocytes of mammalian post injury CNS are considered to be an important player in initiating inflammatory response. The ultrastructure of astroglial cells of injured cord at different time points are as follows: After injury, glial hypertrophy is a key feature in the spinal cord. We have observed increased number of desmosomes between different astroglia in 7-, 10- and 30 dpi cord (Fig. 6 A,B,C). The cells are mostly light astroglia with swollen mitochondria. The cells contain long cisternae of granular endoplasmic reticulum, numerous free ribosomes, rosettes of polyribosomes, many Golgi cisternae, Iysosomes and neuropeptide vesicles (Fig. 6 D,E,F,G,H). Other common features of activated astroglia are increased presence of intermediate filament bundles, dense cytoplasm containing numerous glycogen granules and lysosomes (Figs. 6 B,G, 7 A,B,C). Hypertrophic morphology of astroglia is confirmed by presence of increased extension processes containing many mitochondria and swelling of cell bodies. They are characterized by thick bundles of intermediate filaments after injury (Fig. $7 \mathrm{~A}, \mathrm{~B}$ ). Astrocytic processes are connected by a large number of gap junctions (Figs. $6 \mathrm{~B}, \mathrm{D}, 7 \mathrm{E})$. The length of gap junctions may vary considerably from tiny contact to segments up to $1 \mathrm{~mm}$. These 

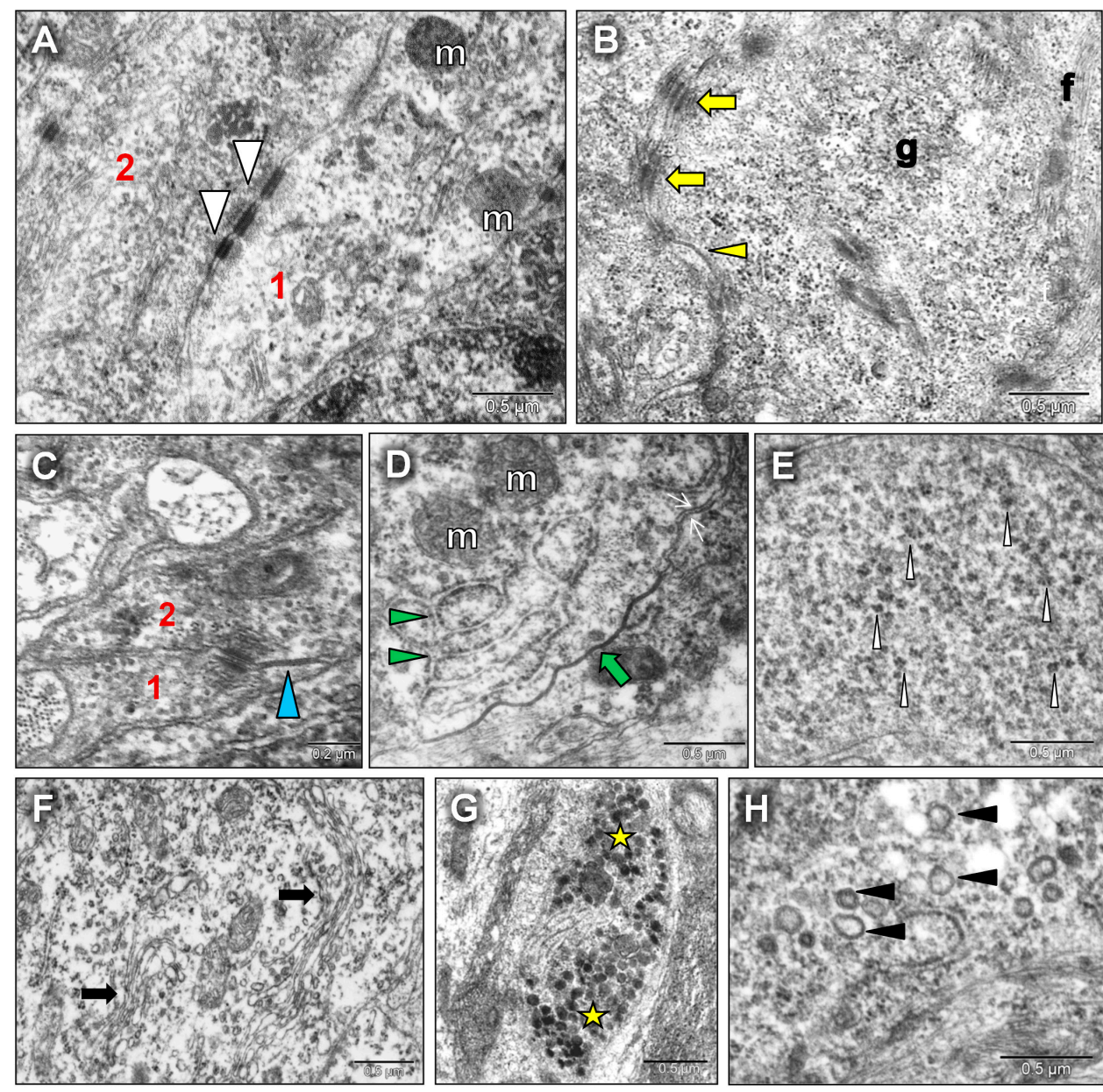

Fig. 6. Ultrastructural features of astroglia in response to injury. (A) TEM image of 7 dpi cord section showing two astroglial cells $(1,2)$, mitochondria ( $m$ ) and are connected by gap junctions (white arrowheads). (B) Section of $10 \mathrm{dpi}$ cord showing many desmosomes (yellow arrows) and one gap junction (yellow arrowhead) in an astroglia replete with glycogen granules (g). ' $f$ ' denotes intermediate filaments. (C) High magnification image showing gap junction (blue arrowhead) between two astroglial processes (1 and 2) is evident. (D) Section of 10 dpi cord showing the cytoplasm of an astroglial cell with long rough endoplasmic reticulum (green arrowheads), swollen mitochondria $(\mathrm{m})$. The same astroglial cell is connected by a long gap junction (green arrow) with another astroglial cell and the plasma membranes (white arrows) at both ends of the cytoplasmic boundary are also visible. (E) 10 dpi cord section showing astroglial cytoplasm is packed with many rosette shaped polyribosomes (white arrowheads). (F) An astroglial cell contains multiple well-developed Golgi bodies (arrows) in the cytoplasm of a 10 dpi cord. (G) An astroglial cytoplasm with many of lysosomal vesicles (yellow star), indicating their known secretary function of growth factors in response to injury. (H) High magnification image of an astroglial cytoplasm in 10 dpi cord showing the presence of many neuropeptide vesicles (arrowheads) in the cytoplasm, probably suggesting their role in neuropeptide mediated signal transmission with adjacent neurons. Scale bar, $0.5 \mu \mathrm{m}(A$, B, D-H); $0.2 \mu \mathrm{m}(\mathrm{C})$. characters are visible in injured cord at different time points (3-, 10-, $15 \mathrm{dpi}$ and even after 1 month of injury). Thus, many intra-astrocytic gap junctions and desmosomes are also present between different astroglia as well as their processes in injured cord (Figs. $6 \mathrm{~A}, 7 \mathrm{~A}, \mathrm{E}$ ). These astroglial processes can also be seen around myelinated axon and immature neurons (Fig. 7E).

All the different cell types involved in injury response and their specific ultrastructural characters are summarized in the Supplementary Table 1. Briefly, in injured spinal cord, astroglial cells showed a nucleus with moderately rich heterochromatin and ample proliferation of glial intermediate filaments in their cytoplasmic processes as well as dark inclusions. The junctional complexes (gap junctions and desmosomes) appeared numerous and extensive between their processes, indicating remodeling after injury due to possible increase in their cytoplasmic area after significant injury-related axonal loss. No significant changes in the features of oligodendrocytes were found in the injured spinal cord, though they appeared somewhat swollen in appearance. Microglias are round-to-oval in shape whereas peripheral macrophages showed amoeboid arms with elongated nuclei, clumps of patchy chromatin near nuclear envelope, relatively dense cytoplasm with rich accumulation of granules, several lysosomes and autophagosomes. Myelin and axonal debris was often encountered in their cytoplasm. They were mostly centered near the site of injury in injured spinal cord than their widespread location in the intact spinal cord of uninjured animals.

\section{Synapse regeneration}

Identification of both excitatory and inhibitory neurons in zebrafish cord has been previously studied (Higashijima et al., 2004a). Based on the neurotransmitter phenotype, neurons can be categorized in larval zebrafish cord. The vesicular glutamate transporter (VGLUT) is present in various glutamatergic neurons that include commissural CoPA, MCoD, UCoD and some of CoSA neurons, whereas cells positive for GLYT2 include CoLA and some of the CoBL and CoSa neurons. GAD expressing neurons are DoLA and KA interneurons. These data provide a map of spinal network active in zebrafish larvae and adult (Higashijima et al., 2004b). We tried to identify some of the types of synapses that are present in regenerating cord at the injury epicenter, based on their morphological characters (Fig. 8).

Generation of different synapses, both axo-axonic or axondendronic synapse can be observed in regenerating cord. A $7 \mathrm{dpi}$ cord shows a hyperactive axo-axonic synapse where presynaptic unit contain both clear and dense core vesicles (Fig. 8A). The regenerated axon terminals usually contain plenty of synaptic vesicles, swollen mitochondria and glycogen granules (Fig. 8B, D). Astroglia are active participants in synapse formation and are very often present around excitatory synapses as seen in Fig 8E. The axoplasm of the injured axons contains glycogen granules and mitochondria (Fig. 8C). Presence of both GABAergic (Fig. 8G) and glutamatergic synapses (Fig. $8 \mathrm{H}$ ) can be demonstrated in regenerated cord 1 month after injury. 


\section{Late regenerative response}

To elucidate the late response to injury, we have chosen different late time points after injury such as 10-, 15- and 30 dpi cord for ultrastructural analysis. In 15 dpi cord, we see presence of both unmyelinated and myelinated axon bundles (Fig. 9A, B). Although at this time point the number of microglia and macrophage present in the injury epicenter is low, phagocytic macrophages play a very important role in clearing toxic myelin debris in earlier stages of regeneration (Fig. 2). Macrophages with engulfed degenerated myelin could still be present in 15 dpi cord, suggesting the importance of macrophages in removal of myelin debris (Fig. 9D). In the late regenerate, we showed that even after 1 month many axons are not myelinated whereas others are myelinated / remyelinated (Fig. 9). At least some of these axons have regenerated since they are present at the injury epicenter and may have been severed during injury. Surprisingly, there is presence of glial progenitors like Schwann cell progenitor(s) in regenerating cord. Role of Schwann cell in remyelination of axons is very remarkable. We observed presence of Schwann cell progenitors, and Schwann cell remyelinating the injured axons (Fig. 9l). We expected to see myelinating oligodendrocytes in the regenerates but so far we have not recorded any oligodendrocyte myelinating damaged (demyelinated) axons, although we found presence of oligodendrocyte progenitors (OPC) in $10 \mathrm{dpi}$ cord (Fig. 5D-E). Identity of these cells was earlier showed to be NG2 positive (Hui et al., 2015).

\section{Discussion}

Spinal cord in zebrafish evokes several cellular responses after injury which is crucial for complete regeneration, yet their causal basis is not well understood. There are some similarities and differences in the cellular response between mammals and fish. In the present study, we used electron microscopy to provide unprecedented view to understand the basis of different cellular events based on the morphological characters and expression of verifiable markers. Thus, ultrastructural analysis facilitates our understanding of different cellular events underlying the extraordinary regeneration process. We have studied the various cellular response(s) after SCl in a time dependentmanner and documented outcome of both early and late events and contribution of several neural progenitors in this remarkable process of CNS regeneration.

\section{Early injury response}

Immediate response after injury is breach of BBB resulting in infiltration of blood cells, cell death which involve both glia and neuron and demyelination of damaged axons. We show initial recruitment of blood borne macrophages in the injury site at very early time points. Although presence of other blood cells like neutrophils and monocytes has been shown in previous analysis (Hui et al., 2010), macrophage remain by far the most important and predominant cell type involved in early injury response and tunes tissue inflammation. The other early response to injury is neuronal cell death. We observed nuclear fragmentation into several highly condensed chromatin bodies,

Fig. 7. Ultrastructure of hyperactive astroglia and astroglia-neuron relationship. (A) TEM image of 10 dpi cord showing astroglia after injury with increased synthesis of glial filaments (f) in astroglial processes that are connected by desmosomes (arrowheads) and gap junctions (arrow). (B) Hypertrophic astroglial processes (stars) in 1 month regenerated cord. (C) $10 \mathrm{dpi}$ cord showing astroglial process with filaments (f) and glycogen (g), swollen mitochondria (m). Few glial processes (stars) with intermediate filaments are also visible. (D) Another 10 dpicord showing a gap junction (black arrow) between two astroglial processes (1, 2), the second astroglial cell (2) has invested the myelinated axon (star). (E) Multiple gap junctions (arrowheads) between two astroglial processes (1, 2). Note presence of one immature neuron (with scanty cytoplasm, star) showing a euchromatic nucleus (n) adjacent to glial process. Scale bar, $0.5 \mu \mathrm{m}$ (A, C, D and E); $1 \mu \mathrm{m}(B)$. 

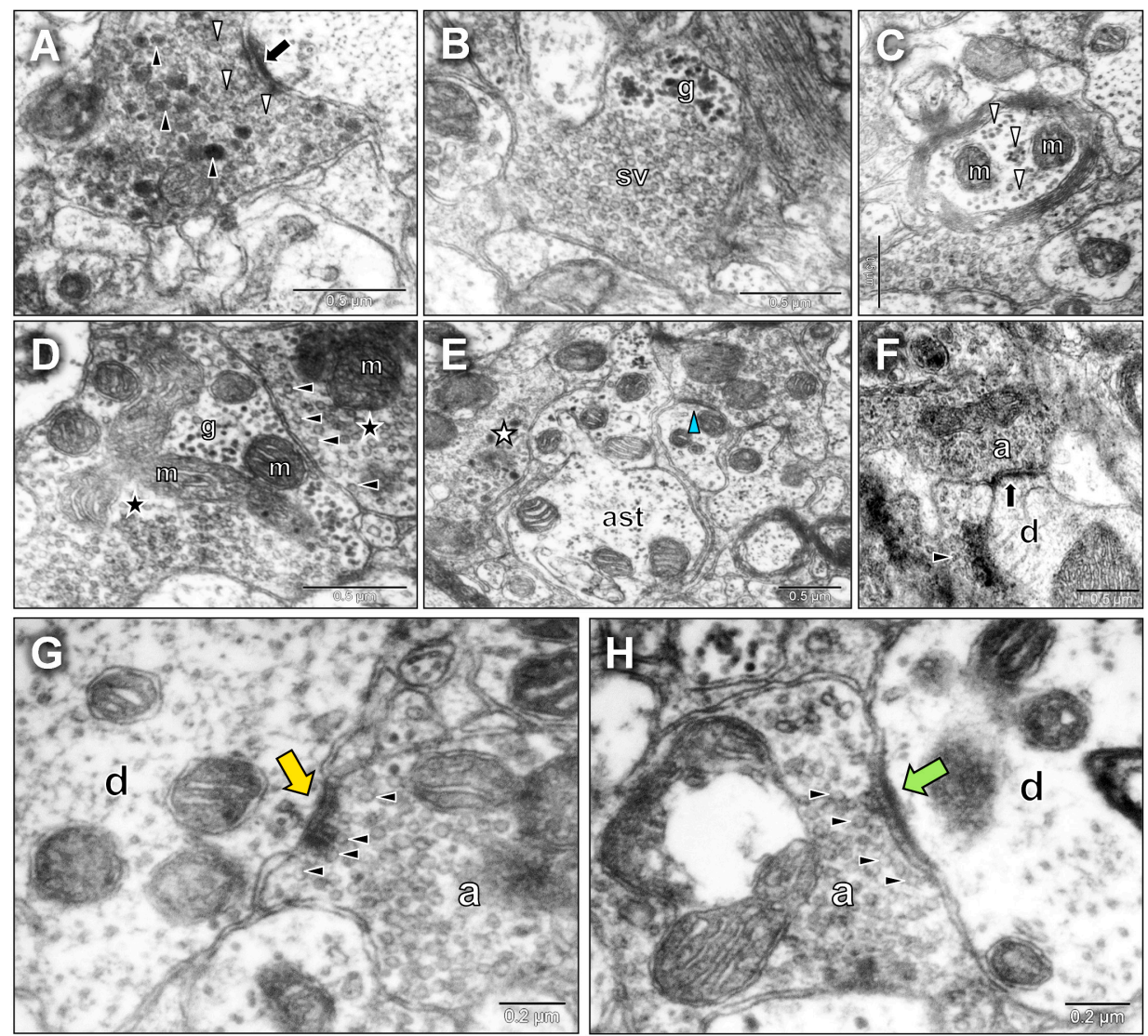

Fig. 8. Ultrastructural features of the regenerating synapse in the spinal cord. (A) TEM image of a 7 dpi cord section showing hyperactive axo-axonic synapse, with presynaptic unit containing clear vesicles (white arrowheads) and few dense-core vesicles (arrowheads), the postsynaptic unit shows neurofilaments (fine black dots) and postsynaptic densities (PSD, arrow). (B) An axon terminal containing clear synaptic vesicles seen as open circles (sv) and numerous glycogen granules (g) in a 1 month regenerate. (C) One month old regenerate showing several axons in cross section, Note the presence of mitochondria ( $m$ ) and glycogen granules (white arrowheads) in the axoplasm. (D) Two axon terminals (stars) with synaptic vesicles (arrowheads), glycogen (g) and mitochondria $(m)$ in 1 month regenerating cord. (E) One month old regenerate showing an excitatory synapse (blue arrowhead), astrocytic process (ast) which is in close contact with a neuronal process containing clear vesicle and dense core vesicle (star). (F) Another 1 month old regenerate showing axon (a) synapsing with a dendrite (d) that shows postsynaptic density (arrow). The same dendrite synapses with a presynaptic

axon showing clusters of vesicles (arrowhead). (G) 1 month old regenerate showing GABAergic synapse (orange arrow) in axo-dendritic (a-d) junction, with many synaptic vesicles (arrowheads). (H) 1 month old regenerate showing glutamatergic synapse (green arrow) in axo-dendritic junction, with many synaptic vesicles (arrowheads) 'a' denotes pre-synaptic axon and 'd' indicates a post-synaptic dendrite. Scale bar, $0.5 \mu \mathrm{m}(A-F) ; 0.2 \mu \mathrm{m}$ (G, H)

shrunken cell cytoplasm often containing irregular mitochondria and having intact infolded cell membrane -the characteristic features of apoptotic neurons. Similar apoptotic cell death has previously been observed in mammalian SCI (Liu et al., 1997; McEwen and Springer, 2005), although much more widespread compared to injured zebrafish cord. Increased presence of macrophages surrounding apoptotic neurons in the injury epicenter in zebrafish cord probably indicates their important role in phagocytosing these dying neurons (Hui et al., 2010). Similar role of resident microglia and peripheral macrophages in clearing damaged and dying neurons has also been reported in zebrafish brain after targeted ablation of neurons (van Ham et al., 2014). Damage to white matter causes loss of glia and myelin. As many axons were severed due to injury, we observe rapid demyelination of damaged axons. During this process, when the myelin sheaths were found to be sloughing off, denuded axons had a very compact array of neurofilaments and increased numbers of mitochondria in the axoplasm compared to the axons of uninjured cord. The blood borne phagocytic macrophages often encroach the demyelinated axons and were involved in devouring the myelin debris, showing lipid filled vacuoles within the cytoplasm. There is a link between macrophage mediated inflammatory response and remyelination, and similar phenomenon seem to be conserved across other species (McMurran et al., 2016). Early inflammatory response is exhibited by microglia and macrophages as they invade injury epicenter very early after injury and play a major role in controlling the detrimental secondary pathophysiological response. In this context, it is important to mention that macrophages express more anti-inflammatory molecules (M2 type genes) other than inflammatory cytokines and remove the toxic breakdown product of myelin after zebrafish $\mathrm{SCl}$ (Hui et al., 2014). As a consequence, macrophages play a major role in removing the effect of myelin derived inhibitory factors and thereby enhance remyelination. Inflammatory response after $\mathrm{SCl}$ in zebrafish cord is brief unlike mammals. Very few microglia and macrophages were present at later time points beyond $10 \mathrm{dpi}$ and if present, the macrophages show engulfed myelin debris and microglia had fewer lysosomes which are known to control inflammation. Macrophage/ microglia may play multiple roles during regeneration and neurogenesis. These cells play important role in promoting neurogenesis in zebrafish brain and spinal cord (Kyritsis et al., 2012; Ohnmatch et al., 2016). Thus, they can be employed in effective reconstruction of neural circuitry by a) providing a niche for generating neurons, b) elimination of unnecessary cells, c) engulfing pruned or degenerating axons and d) involvement in remodeling of synapses (Jin and Yamashita, 2016). Our ultrastructural observation indicated that macrophages during CNS regeneration in zebrafish fulfill many of these roles mentioned above in creating a regeneration permissive niche. 


\section{Proliferative response and involvement of different progenitors}

$\mathrm{SCl}$ invokes early injury response followed by active proliferation of neuronal and glial progenitors in zebrafish cord. In order to replace the lost cells, both glia and neurons, the proliferative response after injury is important and is the key to successful regeneration. Newly born neurons can be seen mostly in $7 \mathrm{dpi}, 10$ $\mathrm{dpi}$ cord and to a lesser extent in $15 \mathrm{dpi}$ and 1 month regenerate, which confirmed the time frame of neurogenesis as mentioned in previous studies (Reimer et al., 2008; Hui et al., 2010; 2015). Our present ultrastructural analysis indicate that there are both early neuronal progenitor (less mature), with high N/C (Nucleus/ Cytoplasmic) ratio and late progenitor (more mature) also with high $\mathrm{N} / \mathrm{C}$ ratio but having the additional presence of dense chromatin in nucleus and few cytoplasmic organelles like mitochondria, RER and active Golgi apparatus. Morphological evidence of neuron-glia interaction represented here are reflective of rearrangement of newly generated neurons in the ventricular zone to its final destination in subependymal zone (Hui et al., 2010). These cells have remarkable
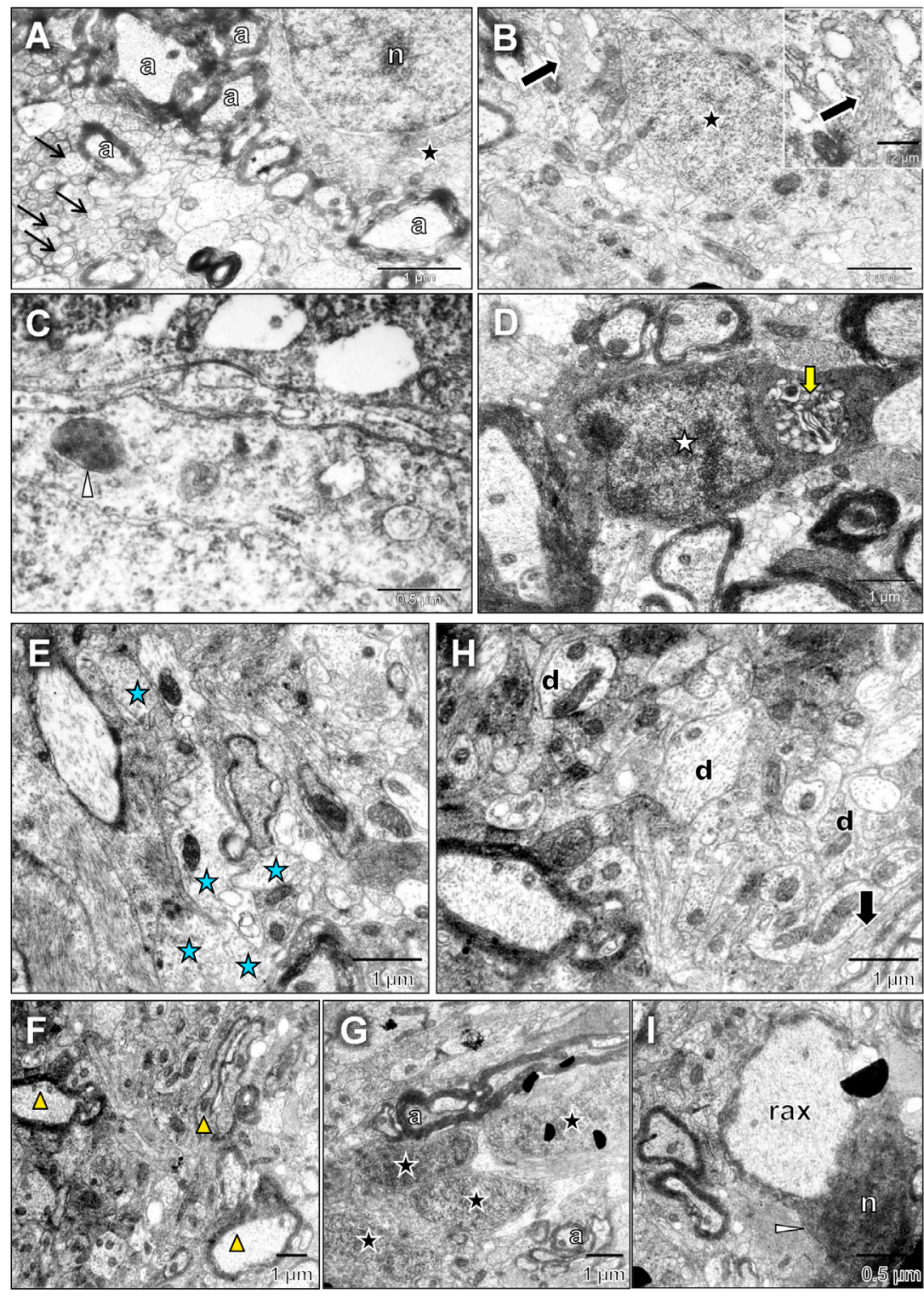

morphological similarity with neuroblasts in mammalian embryonic brain migrating along radial glial fibres and goldfish retina (Rakic, 1971, Lenkowski and Raymond, 2014), although the mechanism and molecular control of neuronal migration during regeneration deserve further analysis. The presence of proliferative progenitors could be observed both in grey matter and white matter. Glial progenitor cells of both astrocytic and oligodendrocytic lineages were present with high N/C ratio. The progenitors of astrocytic lineage had other characters such as glycogen granules and intermediate filaments and glial processes whereas OPC having semi-differentiated cytoplasm with no intermediate filaments, few cisternae of RER and some heterochromatin in nucleus. Some were present around demyelinated axons in white matter. Therefore, the presence of glial restricted progenitors (GRP) has been further confirmed by the ultrastructural analysis. Similarly, identification and characterization of GRP in developing mammalian cord (Rao et al., 1998) also favors the hypothesis that there is creation of an environment similar to embryonic one in regenerating zebrafish cord.

Presence of multiple progenitors have been characterized ultrastructurally and confirmed by their expression of several progenitor markers like Sox2, vimentin and nestin (Lam et al., 2009). Although some of these markers are expressed in uninjured CNS of zebrafish, significant upregulation of these markers in injured cord suggests that different progenitor(s), both glial and neuronal, are generated after injury. These cells include radial glia, OPC, Schwann cell progenitors with increased proliferation and all contribute to generation of lost cells due to $\mathrm{SCl}$.

We also show here that there are increased expressions of progenitor markers like Sox2 and neuronal markers like HuC/D, suggesting more specifically that there are generations of neuronal progenitors in zebrafish cord after injury. This observation is further confirmed by high level of expression of another stem cell marker pou5f1 (mammalian homolog of Oct4) after zebrafish $\mathrm{SCl}$, which is essential for proliferation and neurogenesis (Hui et al., 2014). Other important progenitors contributing to regenerating cord are radial glia, which also express several stem cell markers like Sox2 and vimentin. It has been demonstrated

Fig. 9. Late injury response in the regenerated cord. (A) TEM image of a 15 dpi cord section showing white matter with many unmyelinated axons (black arrows), several myelinated axons (a) and an astroglial cell (star) with a pale nucleus (n). (B) Another 15 day regenerated cord showing an astroglial cell (star) with filaments (arrow). Inset: High magnification view of filaments (arrow). (C,D) 15 day regenerated cord showing $(C)$ a microglial cell with few lysosomes (white arrowhead) and (D) a macrophage (star) with engulfed myelin debris (yellow arrow) in the cytoplasm. (E,F) TEM images of one month regenerated cord showing white matter with many unmyelinated axons ( $E$, blue star) and myelinated axons (F, yellow triangle). (G) Astroglial progenitors (star) and adjacentaxons (a) in the white matter. (H) The neuropil region contains many dendritic profiles (d) and an unidentified cell process (arrow). (I) The nucleus (n) of a Schwann cell and with the basal membrane (arrowhead) is wrapping around the remyelinating axon (rax). Scale bar, $1 \mu \mathrm{m}(A, B, D, E, F, G$ and $H) ; 0.5 \mu \mathrm{m}$ (C and $E)$. 
that sustained expression of Sox2 confers the progenitors to regenerative mode as observed in Müllerian glia in zebrafish and medaka retina (Ramachandran, 2010) and similarly radial glia in zebrafish spinal cord (Hui et al., 2014, 2015; Ogai et al., 2014) express Sox2 conferring plasticity to these cells.

\section{Astroglial response to injury}

In zebrafish, radial glia persist throughout life, whereas in mammals they are found in developing CNS and are later transformed into astrocytes (Rakic, 2003; Malatesta et al., 2008). The presence of stellate astrocyte in zebrafish CNS is debatable. Although some reported absence of stellate astrocytes in the zebrafish brain (Grupp et al., 2010) others showed presence of small astrocytes with no axons (Kawai et al., 2001) in spinal cord. Lack of parenchymal astrocytes and presence of radial glia as the major form of astroglia in zebrafish CNS is confirmed by the present analysis, as reported by others (Hui et al., 2010; Briona and Dorsky, 2014; Becker and Becker, 2015; Becker et al., 2018). Furthermore, radial glia are spinal neural stem cells known to generate both neuron and glia, and many of these are at a quiescent stage (Kriegstein and Alvarez- Buyella, 2009; Hui et al., 2010; Malatesta and Gotz, 2013; Becker and Becker, 2015; Becker et al., 2018).

The zebrafish spinal cord has unique response to injury where radial glia exhibit both gliotic and regenerative potential. Understanding and manipulating both gliotic changes and regenerative properties of the progenitor(s) would allow us to generate a therapeutic potential for $\mathrm{SCl}$ in higher vertebrates. Astrocytes in mammals support neuronal function by promoting synaptic contact thereby regulating the flow of ions and neurotransmitters and hence providing metabolic substrate for neurons and maintaining BBB (Sun and Jakobs, 2012). Secondary injury response in CNS involves astrocytes, which play a key role in reuptake of glutamate from synapses to protect neurons which are highly vulnerable to excitotoxicity (Palmer et al., 1993). Mammalian CNS injury reactions involve microglia, astrocytes and OPC. Astrocytes contribute to neuropathology through astrogliosis when they react to injury by hypertrophy of somata and show increased synthesis of GFAP, re-expression of other markers like nestin and vimentin (Bignami and Dahl, 1976; Sofreniew, 2005; Dimou and Gotz, 2014). Based on the diverse response of astroglia after injury and in disease conditions, two functionally distinct categories of reactive astrocytes have been proposed, namely scar forming reactive astrocytes and hypertrophic reactive astrocytes (Sofroniew, 2009; Khakh and Sofroniew, 2015). Here astroglial cells in zebrafish show hypertrophy after injury, yet are not forming scar and may be involved in protecting neurons and synapses.

In zebrafish CNS, injury response involves limited wound size and inflammation yet no formation of astroglial scar. We show some feature of gliosis after SCI, like marked glial hypertrophy, cell cycle re-entry and increased expression of various markers like vimentin, glutamine synthetase (GS), GFAP in radial glial cells (Hui et al., 2010, 2015). Similar to mammalian reactive Müllerian glia, where these cells may retain their normal metabolic function even when proliferating (Vazquez-chona et al., 2011), zebrafish radial glia may undergo dedifferentiation (Hui et al., 2015) but retain normal glial homeostasis. The observed features of gliosis after zebrafish $\mathrm{SCl}$ are similar to that in mammals. Our ultrastructural analysis confirmed the features of gliosis. Many of these features are also observed in other fish (Bernstein and Bernstein, 1967;
Bignami et al., 1974; Kalman, 2004).

Present analysis revealed presence of hypertrophic swollen astroglia with conspicuous nucleoli and many processes containing increased cellular organelles, like mitochondria, Golgi bodies with more cisternae and RER. Several other features observed are increased content of intermediate filaments and glycogen granules and many junctional complexes. These features may be suggestive of their contribution to the energy metabolism of neurons (Koizumi, 1974; Magistretti et al., 1993). Both hypertrophic astroglia in injured cord and normal astroglia in uninjured cord are connected to each other by desmosomes and gap junctions, similar to what has been observed in mature mammalian astrocytes. Presence of neuropeptide vesicles in astroglia also indicates their role in neurotransmitter recycling. Association of these cells around excitatory synapses may point towards their function in the maintenance of synapses.

Unlike mammals, zebrafish have remarkable capacity to regenerate neurons in many parts of the CNS. As mentioned previously, the radial glia act as stem cells to generate appropriate progenitors for regeneration (Reimer et al., 2008; Briona and Dorsky, 2014; Hui et al., 2015; Becker and Becker, 2015; Becker et al., 2018). Under normal conditions, these glia are responsible for support and maintenance of neurons as they regulate glucose metabolism and control recycling of the neurotransmitter glutamate, blood flow and water homeostasis (Nagelhus et al., 1998; Grupp et al., 2010; Kettenmann and Ransom, 2012). These cells are indispensable for GABAergic and glutamatergic synaptic transmission as they control the supply of neurotransmitter precursor molecules (Verkhratsky et al., 2015). Neurotransmitter mediated regeneration of zebrafish retina (Rao et al., 2017) involve Müllerian glia and inhibition of GABA signaling facilitate Müllerian glia proliferation. Whereas dopamine and serotonin from brain induce spinal motor neuron regeneration in zebrafish (Reimer et al., 2013, Barreiro-Iglesias et al., 2015) by modulating regenerative neurogenesis. We observed successful regeneration of GABAergic and glutamatergic synapses and glia-neuronal interaction is also evident.

\section{Late response to injury}

The late response during regeneration consists of regrowth and remyelination of axons. One of the injury responses is denudation of damaged axons that we have seen immediately after injury. Ultrastructural characters shown here include extensive degenerative changes with many damaged axons containing very dense neurofilaments and ample mitochondria. The role of mitochondria in controlling the oxidative energy metabolism during CNS regeneration has not been elucidated and needs future attention. There is functional recovery after $\mathrm{SCl}$ in zebrafish, although not all the damaged axons can regrow and remyelinate. The volume of white matter in regenerated cord does not reach $100 \%$ (Hui et al., 2010). Thickness of myelin sheath in remyelinated axons are always less compared to uninjured axons, as confirmed by G-ratio. Schwann cells, the PNS glia, are highly versatile and may perform similar function like myelin formation, synaptic junction ensheathing, etc in zebrafish CNS. We confirmed the presence of Schwann cell progenitors and involvement of these cells in remyelination as shown by Nona et al., (1992) during goldfish optic nerve regeneration. Although synapse regeneration is important in rescuing neuronal function after $\mathrm{SCl}$, very little is known about the structures and molecular identity of regenerated synapse. There are some reports on synaptic regeneration in various other species in 
lampreys, leech and Drosophila (Miladinic et al., 2009; Oliphint et al., 2010) showing that regenerating CNS axons form appropriate synapses. Our data represent regeneration of different types of synapses such as GABAergic and glutamatergic synapses. We locate ultrastructure of these structures in injury epicenter, in particular GABAergic synapse of Kolmer-Agduhr interneurons, which are located near the ependyma, as observed by others (Bernhardt et al., 1992). However, more exhaustive quantitative analysis like number, axonal distribution and molecular composition along with ultrastructural features need to be studied in this species. We showed basic structural characteristic of regenerated synapses that may have contributed to functional recovery after SCl.

In conclusion, we suggest that there should be a combinatorial approach to understand the injury response in the zebrafish CNS. Future studies may address and emphasize on uncovering various aspects viz. a) the specific role of neuronal as well glial progenitors in generating new neurons and glia, b) in-depth analysis of the role of macrophages in controlling deleterious role of neuroinflammation and enhancement of regeneration, c) how the beneficial role of astrogliosis could be augmented and d) elucidating the molecular and cellular control underlying induction of axonal regrowth and remyelination. Here, we gave a most comprehensive ultrastructural account of different cellular events during regeneration of zebrafish cord, which may shed more light on our present understanding and generating therapeutic strategies. However, more attention has to be given to study the intricate details of intrinsic regeneration capability in lower vertebrate models like urodeles and teleost fish in order to successfully induce spinal cord regeneration in higher vertebrates.

\section{Materials and Methods}

All animal experiments mentioned in this study were cleared by Institutional Animal Ethics Committee, Department of Biophysics, Molecular Biology and Bioinformatics, University of Calcutta, Kolkata under CPCSEA registration of Govt. of India (Registration Number CPCSEA/ORG/CH/ Reg. No 925/295).

\section{Zebrafish maintenance}

Adult zebrafish of both sexes with age ranging from of 4-6 months were maintained in the Aquatic system in the animal house facility with a $14 \mathrm{hr}$ light/10 hr dark cycle. They were housed in separate groups of 10-12 in each tank and maintained at a constant water temperature of $28^{\circ} \mathrm{C}$ along with a standard feeding regime.

\section{Surgical procedures}

Adult fishes were anaesthetized for $5 \mathrm{~min}$ in $0.02 \%$ tricaine methanesulfonate (MS222; Sigma-Aldrich Corporation, St. Louis, MO) before performing any surgical procedures. Spinal cord injury was performed as described in Hui and Ghosh (2016). Briefly, a skin incision was made laterally at the level of the dorsal fin to expose the vertebral column, which corresponds to the 15-16th vertebrae as confirmed previously (Hui et al., 2010). The spinal cord was crushed dorso-ventrally for about $1 \mathrm{sec}$ with a number-5 Dumont forceps. A fine suture was used to close the muscle and cutaneous wound and fishes were immediately transferred to aquarium water and allowed to regenerate at $28^{\circ} \mathrm{C}$. Regenerating spinal cord tissues (from 5-8 samples) at different time points viz. 3 day post injury (dpi), 7 $\mathrm{dpi}, 10 \mathrm{dpi}, 15 \mathrm{dpi}$ and $30 \mathrm{dpi}$ were collected after terminal anaesthesia was applied to the fishes and only spinal cord tissues were dissected out by opening the bony encasing. About $2 \mathrm{~mm}$-length of spinal cord including the regenerating tissue with adjacent uninjured tissue on either side was excised from the whole spinal cord.

\section{Transmission electron microscopy}

Both injured and uninjured spinal cord tissues were immediately fixed in $2 \%$ paraformaldehyde and $2.5 \%$ glutaraldehyde in $0.1 \mathrm{M}$ phosphate buffer (PB, $\mathrm{pH} \mathrm{7.3)} \mathrm{for} 1 \mathrm{~h}$ at room temperature and for another $18 \mathrm{hs}$ in the same fixative at $4^{\circ} \mathrm{C}$. After washing with buffer, tissues were osmicated in $1 \% \mathrm{OsO}_{4}$ in $0.1 \mathrm{M}$ phosphate buffer and embedded in Araldite CY212. Ultrathin sections (70-80 nm) were cut using an ultramicrotome (Leica, Wetzlar, Germany) and stained with uranyl acetate and lead citrate and viewed under a Morgagni 268D electron microscope (FEl Company, Eindhoven, The Netherlands). Data represented here come from analysis of at least three tissue samples at any particular time point. Time course analysis of regeneration was performed using 5 different time points of injury, along with uninjured cord.

\section{Immunohistochemistry}

Immunostaining was performed as reported earlier (Hui et al., 2010) by using primary antibodies shown to specifically recognize zebrafish. Briefly, sections were rehydrated and rinsed in phosphate buffered saline (PBS) with $0.1 \%$ Tween-20 (PBSTX). Then sections were incubated with blocking solution for $1 \mathrm{hr}(5 \% \mathrm{goat} / \mathrm{rabbit} /$ donkey serum, $1 \% \mathrm{BSA}$ in PBS) at room temperature and after that with primary antibody for overnight at $4^{\circ} \mathrm{C}$. All primary antibodies were diluted in PBSTX. To unmask some antigens in paraformaldehyde fixed tissues, antigen retrieval has been performed by incubating the slides in $95^{\circ} \mathrm{C}$ warm water bath for 15 mins in sodium-citrate buffer ( $\mathrm{pH}$ 6.0) before incubation with the blocking buffer. The following primary and secondary antibodies were used for this study: Anti-glial fibrillary acidic protein (GFAP; dilution: 1:500, DAKO, USA, Catalogue no. 20334); Anti-BrdU (dilution: 1:300, Sigma, USA, Catalogue no. B-8434); Anti-brain lipid binding protein (BLBP; dilution: 1:400, Abcam PIc, USA, Catalogue no. Ab-27171); Anti-glutamate aspartate transporter (GLAST; dilution: 1:400, Chemicon, USA, Catalogue no. AB1782); Anti-vimentin (dilution: 1:300, Millipore, USA, Catalogue no. MAB3400); Anti-Sox2 (dilution: 1:400, Abcam USA, Catalogue no. Ab15830); Anti-HuC/D (dilution: 1:50, Molecular Probes, USA, Catalogue no. A-21271); TRITC-conjugated goat anti-rabbit IgGantibody (dilution: 1:400, Jackson ImmunoResearch Laboratories, USA); FITC-conjugated goat antirabbit IgG antibody (dilution: 1:400, Jackson ImmunoResearch Laboratories, USA); FITC-conjugated goat anti-mouse (dilution: 1:200, Santa Cruz Biotechnology, CA, USA); TRITC-conjugated goat anti-mouse (dilution: 1:400, Jackson ImmunoResearch Laboratories, USA). Nuclei were stained either with 4',6-diamidino-2-phenylindole (DAPI) or with Hoechst (Sigma, USA). BrdU immunohistochemistry was performed according to previous communication (Hui et al., 2010).

\section{Quantitative reverse transcription polymerase chain reaction (qRT- PCR)}

Total RNA was extracted using TRIzol reagent, and cDNA was subsequently synthesized either with the Transcriptor first strand cDNA synthesis kit (Roche, Basel, Switzerland) or SensiFAST cDNA Synthesis Kit (Bioline, Australia). qRT-PCR was performed using a LightCycler 480 system (Roche, Basel, Switzerland). The amount of cDNA was normalized according to actb2 amplification in qRT-PCR. All qRT-PCR were performed using SYBR ${ }^{\mathrm{TM}}$ Select Master Mix (Applied Biosystems, CA, USA) and results obtained from at least 5 biological replicates. The fold induction was calculated by setting control conditions to 1 . The following primer sequences were used for the qRT-PCR analysis vim: Forward [5'- aggaggccaatgattaccg -3'], Reverse [5'- acgctccagagactcattcg -3']; gfap: Forward [5'- aaatcgccacctacaggaag -3'], Reverse [5'- tctgcaccggaacagtgat -3']; nes: Forward [5'- agaccaacaccaaacaagaccag -3'], Reverse [5'- acacagaactgctgagaaggca -3'].

\section{Acknowledgements}

This work is supported by DST (Govt. of India), and UGC UPEII program grant (University of Calcutta) to SG. SPH performed all experiments, TCN, SG and SPH analyzed the data and SG, SPH had written the manuscript. All authors read the final draft of the manuscript. We would like to thank Debojyoti De for compiling bibliography section. 


\section{References}

ADOLFB, CHAPOUTON P, LAM CS, TOPP S, TANNHÄUSERB, STRÄHLEU, GÖTZ M, BALLY-CUIF L (2006). Conserved and acquired features of adult neurogenesis in the zebrafish telencephalon. Dev Biol 295: 278-293.

ALUNNI ALUNNI A, VACCARI S, TORCIA S, MEOMARTINI ME, NICOTRA A, ALFEI L (2005). Characterization of glial fibrillary acidic protein and astroglial architecture in the brain of a continuously growing fish, the rainbow trout. Eur $J$ Histochem 49: 157-166.

BALENTINE JD (1978a). Pathology of experimental spinal cord trauma. I. The necrotic lesion as a function of vascular injury. Lab Invest 39: 236-53.

BALENTINE JD (1978b). Pathology of experimental spinal cord trauma. II. Ultrastructure of axons and myelin. Lab Invest 39: 254-266.

BARREIRO-IGLESIAS A, MYSIAK KS, SCOTT AL, REIMER MM, YANG Y, BECKER CG, BECKER T (2015). Serotonin Promotes Development and Regeneration of Spinal Motor Neurons in Zebrafish. Cell Rep 13: 924-932.

BECKER CG, BECKER T, HUGNOT JP (2018). The spinal ependymal zone as a source of endogenous repair cells across vertebrates. Prog Neurobiol 170: 67-80.

BECKER CG, BECKER T (2015). Neuronal regeneration from ependymo-radial glial cells: cook, little pot, cook! Dev Cell 32: 516-527.

BERNHARDT RR, PATEL CK, WILSON SW, KUWADAJY (1992). Axonal trajectories and distribution of GABAergic spinal neurons in wildtype and mutant zebrafish lacking floor plate cells. J Comp Neurol 326: 263-272.

BERNSTEIN JJ, BERNSTEIN ME (1967). Effect of glial-ependymal scar and teflon arrest on the regenerative capacity of goldfish spinal cord. Exp Neurol 19: 25-32.

BIGNAMIA, DAHLD (1976). The astroglial response to stabbing. Immunofluorescence studies with antibodies to astrocyte-specific protein (GFA) in mammalian and submammalian vertebrates. Neuropathol Appl Neurobiol 2: 99-110.

BIGNAMI A, FORNO L, DAHL D (1974). The neuroglial response to injury following spinal cord transection in the goldfish. Exp Neurol 44: 60-70.

BLIGHTAR (1985). Delayed demyelination and macrophage invasion: a candidate for secondary cell damage in spinal cord injury. Cent Nerv Syst Trauma 2: 299-315.

BRIONA LK, DORSKY RI (2014). Radial glial progenitors repair the zebrafish spinal cord following transection. Exp Neurol 256: 81-92.

BUFFO A, RITE I, TRIPATHI P, LEPIER A, COLAK D, HORN AP, MORI T, GÖTZ M (2008). Origin and progeny of reactive gliosis: A source of multipotent cells in the injured brain. Proc Natl Acad Sci USA 105: 3581-3586.

DIMOU L, GÖTZ M (2014). Glial cells as progenitors and stem cells: new roles in the healthy and diseased brain. Physiol Rev 94:709-737.

FAULKNER JR, HERRMANN JE, WOO MJ, TANSEY KE, DOAN NB, SOFRONIEW MV (2004). Reactive astrocytes protect tissue and preserve function after spinal cord injury. J Neurosci 24: 2143-2155.

GOLDSHMIT Y, SZTAL TE, JUSUF PR, HALL TE, NGUYEN-CHI M, CURRIE PD (2012). Fgf-dependent glial cell bridges facilitate spinal cord regeneration in zebrafish. J Neurosci 32: 7477-7492.

GRUPP L, WOLBURG H, MACK AF (2010). Astroglial structures in the zebrafish brain. J Comp Neurol 518: 4277-4287.

GUTH L, ZHANG Z, STEWARD O (1999). The unique histopathological responses of the injured spinal cord: implications for neuroprotective therapy. Ann NY Acad Sci 890: 366-384

HIGASHIJIMA S, MANDEL G, FETCHO JR (2004a). Distribution of prospective glutamatergic, glycinergic, and GABAergic neurons in embryonic and larval zebrafish. J Comp Neurol 480: 1-18.

HIGASHIJIMA S, MANDEL G, FETCHO JR (2004b). Neurotransmitter properties of spinal interneurons in embryonic and larval zebrafish. J Comp Neurol 480: 19-37.

HUI SP, DUTTA A, GHOSH S (2010). Cellular response after crush injury in adult zebrafish spinal cord. Dev Dyn 239: 2962-2979.

HUI SP, GHOSH S (2016). Various Modes of Spinal Cord Injury to Study Regeneration in Adult Zebrafish. Bio-protocol 6: e2043.

HUISP, NAG TC, GHOSHS (2015). Characterization of proliferating neural progenitors after spinal cord injury in adult zebrafish. PLoS One 10: e0143595.

HUI SP, SENGUPTAD, LEE SG, SEN T, KUNDU S, MATHAVAN S, GHOSH S (2014). Genome wide expression profiling during spinal cord regeneration identifies comprehensive cellular responses in zebrafish. PLoS One 9: e84212.
ITOY, TANAKAH, OKAMOTO H, OHSHIMAT (2010). Characterization of neural stem cells and their progeny in the adult zebrafish optic tectum. Dev Bio/ 342: 26-38.

JIN X, YAMASHITA T (2016). Microglia in central nervous system repair after injury. $J$ Biochem 159: 491-496.

JOHANSSON CB, MOMMA S, CLARKE DL, RISLING M, LENDAHL U, FRISÉN J (1999). Identification of a neural stem cell in the adult mammalian central nervous system. Cell 96: 25-34.

KALMAN M (2004). Glial reaction and reactive glia. Adv Mol Cell Biol 31: 787-835.

KAWAI H, ARATA N, NAKAYASU H (2001). Three-dimensional distribution of astrocytes in zebrafish spinal cord. Glia 36: 406-413.

KETTENMANN H, BRUCE RR (2012). Neuroglia $3^{\text {rd }}$ ed. New York, Oxford University Press. p. 864.

KHAKHBS, SOFRONIEWMV (2015). Diversity of astrocyte functions and phenotypes in neural circuits. Nat Neurosci 18: 942-952.

KOIZUMI J (1974). Glycogen in the central nervous system. Prog Histochem Cytochem 6: 1-35.

KRIEGSTEINA, ALVAREZ-BUYLLAA (2009). The glial nature of embryonic and adult neural stem cells. Annu Rev Neurosci 32: 149-184.

KYRITSIS N, KIZIL C, ZOCHER S, KROEHNE V, KASLIN J, FREUDENREICH D, ILTZSCHE A, BRAND M (2012). Acute inflammation initiates the regenerative response in the adult zebrafish brain. Science 338: 1353-1356.

LAM CS, MÄRZ M, STRÄHLE U (2009). GFAP and nestin reporter lines reveal characteristics of neural progenitors in the adult zebrafish brain. Dev Dyn238: 475-486.

LENKOWSKI JR, RAYMOND PA (2014). Müller glia: Stem cells for generation and regeneration of retinal neurons in teleost fish. Prog Ret Eye Res 40: 94-123.

LIU XZ, XU XM, HU R, DU C, ZHANG SX, MCDONALD JW, DONG HX, WU YJ, FAN GS, JACQUIN MF, HSU CY, CHOI DW (1997). Neuronal and glial apoptosis after traumatic spinal cord injury. J Neurosci 17: 5395-5406.

MAGISTRETTI PJ, SORG O, MARTIN JL (1993). Regulation of glycogen metabolism in astrocytes: physiological, pharmacological, and pathological aspects. Astrocytes: pharmacology and function. Academic Press, San Diego. pp. 243-265.

MALATESTA P, APPOLLONI I, CALZOLARI F (2008). Radial glia and neural stem cells. Cell Tissue Res 331: 165-178.

MALATESTAP, GÖTZM (2013). Radial glia-from boring cables to stem cell stars. Development 140:483-486.

MCEWEN ML, SPRINGER JE (2005). A mapping study of caspase-3 activation following acute spinal cord contusion in rats. J Histochem Cytochem 53: 809-819.

MCMURRAN CE, JONES CA, FITZGERALD DC, FRANKLIN RJ (2016). CNS Remyelination and the Innate Immune System. Front Cell Dev Biol 4: 38.

MLADINIC M, MULLER KJ, NICHOLLS JG (2009). Central nervous system regeneration: from leech to opossum. J Physiol 587: 2775-82.

NAGELHUS EA, VERUKI ML, TORP R, HAUG FM, LAAKE JH, NIELSEN S, AGRE P, OTTERSEN OP (1998). Aquaporin-4 water channel protein in the rat retina and optic nerve: polarized expression in Müller cells and fibrous astrocytes. $J$ Neurosci 18: 2506-2519.

NONASN, DUNCANA, STAFFORD CA, MAGGSA, JESERICH G, CRONLY-DILLON JR (1992). Myelination of regenerated axons in goldfish optic nerve by Schwann cells. J Neurocytol 21: 391-401.

OLIPHINT PA, ALIEVA N, FOLDES AE, TYTELL ED, LAU BY, PARISEAU JS, COHEN AH, MORGAN JR (2010). Regenerated synapses in lamprey spinal cord are sparse and small even after functional recovery from injury. $J$ Comp Neurol 518: 2854-2872.

PALMER AM, MARION DW, BOTSCHELLER ML, SWEDLOW PE, STYREN SD, DEKOSKY ST (1993). Traumatic brain injury-induced excitotoxicity assessed in a controlled cortical impact model. J Neurochem 61: 2015-2024.

PETERS A, PALAY SL, WEBSTER H (1991). The fine structure of the nervous system: neurons and their supporting cells. $3^{\text {rd }}$ ed, Oxford University Press, London.

POPOVICH PG, WEI P, STOKES BT (1997). Cellular inflammatory response after spinal cord injury in Sprague-Dawley and Lewis rats. J Comp Neuro/377: 443-464.

RAKIC P (1971). Neuron-glia relationship during granule cell migration in developing cerebellar cortex. A Golgi and electronmicroscopic study in Macacus Rhesus. J Comp Neurol 141: 283-312.

RAKIC P (2003). Elusive radial glial cells: historical and evolutionary perspective. Glia 43: 19-32. 
RAMACHANDRAN R, FAUSETT BV, GOLDMAN D (2010). Ascl1a regulates Müller glia dedifferentiation and retinal regeneration through a Lin-28-dependent, let-7 microRNA signalling pathway. Nat Cell Biol 12: 1101-1107.

RAOMB, DIDIANOD, PATTON JG (2017). Neurotransmitter-Regulated Regeneration in the Zebrafish Retina. Stem Cell Reports 8: 831-842.

RAO MS, NOBLE M, MAYER-PRÖSCHEL M (1998). A tripotential glial precursor cell is present in the developing spinal cord. Proc Natl Acad Sci USA 95: 3996-4001.

REIMER MM, NORRIS A, OHNMACHT J, PATANI R, ZHONG Z, DIAS TB, KUSCHA V, SCOTT AL, CHEN YC, ROZOV S, FRAZER SL, WYATT C, HIGASHIJIMA S, PATTON EE, PANULA P, CHANDRAN S, BECKER T, BECKER CG (2013). Dopamine from the brain promotes spinal motor neuron generation during development and adult regeneration. Dev Cell 25: 478-491.

REIMER MM, SÖRENSEN I, KUSCHA V, FRANK RE, LIU C, BECKER CG, BECKER $\mathrm{T}$ (2008). Motor neuron regeneration in adult zebrafish. J Neurosci28: 8510-8516.

RUDGE JS, SILVER J (1990). Inhibition of neurite outgrowth on astroglial scars in vitro. J Neurosci 10: 3594-3603.

SILVER DJ, STEINDLER DA (2009). Common astrocytic programs during brain development, injury and cancer. Trends Neurosci 32: 303-311.

SILVER J, MILLER JH (2004). Regeneration beyond the glial scar. Nat Rev Neurosci 5: 146.

SILVERJ (2016). The glial scar is more than just astrocytes. Exp Neurol286: 147-149.

SOFRONIEW MV (2005). Reactive astrocytes in neural repair and protection. Neu- roscientist 11: 400-407.

SOFRONIEW MV (2009). Molecular dissection of reactive astrogliosis and glial scar formation. Trends Neurosci 12: 638-647.

SUN D, JAKOBS TC (2012). Structural remodeling of astrocytes in the injured CNS. Neuroscientist 18: 567-588.

TEMPLE S, ALVAREZ-BUYLLAA (1999). Stem cells in the adult mammalian central nervous system. Curr Opin Neurobiol 9: 135-141.

VAN HAM TJ, BRADY CA, KALICHARAN RD, OOSTERHOF N, KUIPERS J, VEENSTRA-ALGRA A, SJOLLEMA KA, PETERSON RT, KAMPINGA HH, GIEPMANS BN (2014). Intravital correlated microscopy reveals differentia macrophage and microglial dynamics during resolution of neuroinflammation. Dis Model Mech 7: 857-869.

VÁZQUEZ-CHONA FR, SWAN A, FERRELL WD, JIANG L, BAEHR W, CHIEN WM FERO M, MARC RE, LEVINE EM (2011). Proliferative reactive gliosis is compatible with glial metabolic support and neuronal function. BMC Neurosci 12: 98.

VERKHRATSKY A, NEDERGAARD M, HERTZ L (2015). Why are astrocytes important? Neurochem Res 40: 389-401.

YAMADA H, MIYAKE T, KITAMURA, T (1997). Proliferation and differentiation of ependymal cells after transection of the carp spinal cord. Zoological science 14: 331-339.

ZUPANC GK, HINSCH K, GAGE FH (2005). Proliferation, migration, neuronal differentiation, and long-term survival of new cells in the adult zebrafish brain. $J$ Comp Neurol 488: 290-319. 


\section{Further Related Reading, published previously in the Int. J. Dev. Biol.}

Transplantation of Xenopus laevis ears reveals the ability to form afferent and efferent connections with the spinal cord Karen L. Elliott and Bernd Fritzsch

Int. J. Dev. Biol. (2010) 54: 1443-1451

Oligodendrocyte development in the embryonic brain: the contribution of the plp lineage

Barbara Le Bras, Elli Chatzopoulou, Katharina Heydon, Salvador Martínez, Katzuhiko Ikenaka, Laetitia Prestoz, Nathalie Spassky, Bernard Zalc and Jean-Léon Thomas

Int. J. Dev. Biol. (2005) 49: 209-220

Distinct neural precursors in the developing human spinal cord

Sally Walder and Patrizia Ferretti

Int. J. Dev. Biol. (2004) 48: 671-674

Notch activity is required to maintain floorplate identity and to control neurogenesis in the chick hindbrain and spinal cord. Isabelle le Roux, Julian Lewis and David Ish-Horowicz Int. J. Dev. Biol. (2003) 47: 263-272

Patterning of the vertebrate ventral spinal cord.

Alisa Poh, Asanka Karunaratne, Gabriel Kolle, Ning Huang, Emma Smith, Joanna Starkey, Daying Wen, Ian Wilson, Toshiya Yamada and Murray Hargrave

Int. J. Dev. Biol. (2002) 46: 597-608

From hematopoietic stem cells to neural stem cells

S Bonilla, P Alarcon, R Villaverde, P Aparicio, A Silva, S Martinez

Int. J. Dev. Biol. (2001) 45: S67-S68

Astroglia-microvessel relationship in the developing human telencephalon.

D Virgintino, E Maiorano, M Errede, A Vimercati, P Greco, LSelvaggi, L Roncali and M Bertossi Int. J. Dev. Biol. (1998) 42: 1165-118

Astroglial differentiation from neuroepithelial precursor cells of amphibian embryos: an in vivo and in vitro analysis.

C Soula, Y Sagot, P Cochard and A M Duprat

Int. J. Dev. Biol. (1990) 34: 351-364
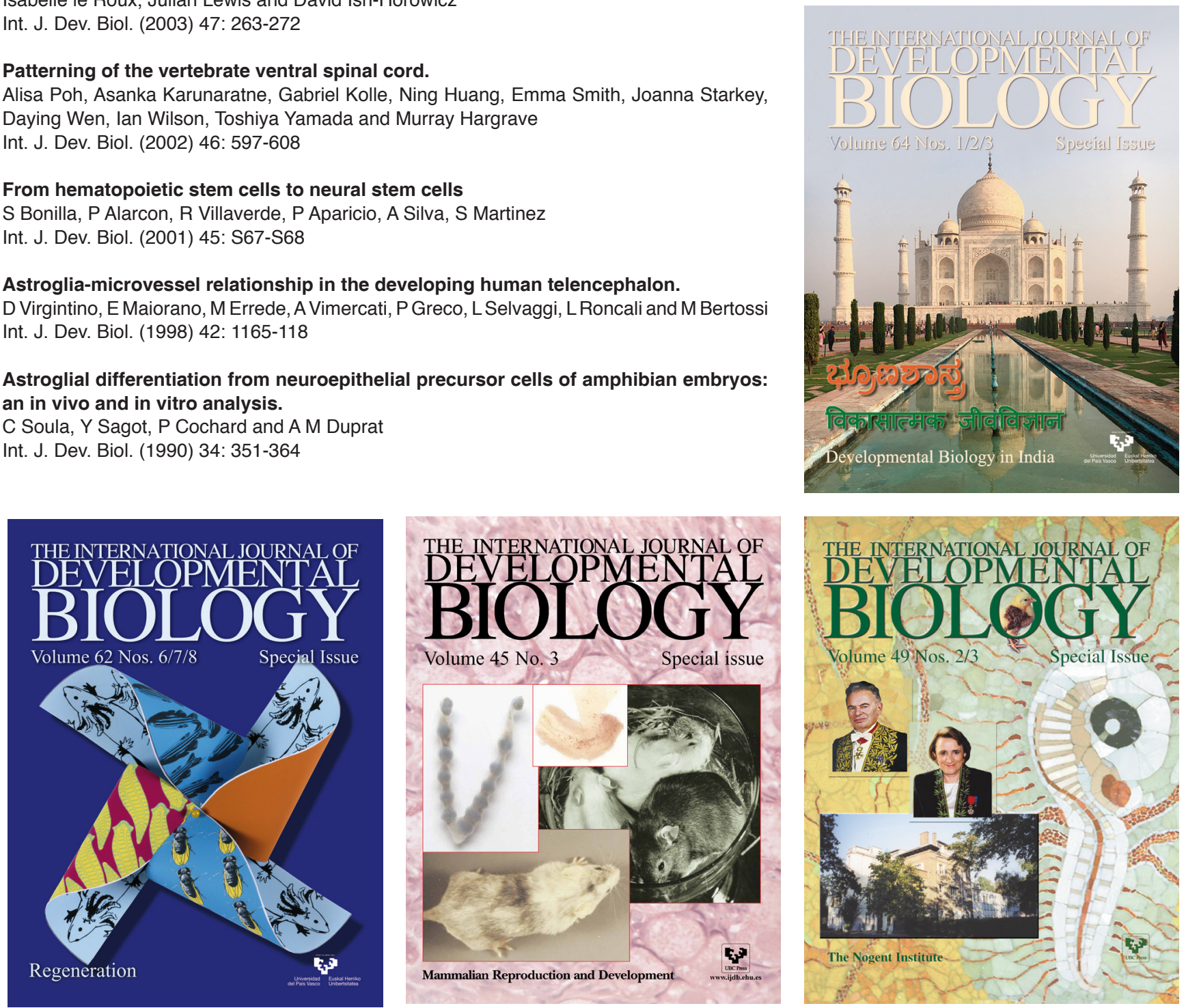